\title{
Geração de energia elétrica através de Sistemas Fotovoltaicos para consumidores comerciais de pequeno porte
}

\author{
Electric power generation through photovoltaic systems for small commercial consumers
}

\author{
Gabriela Pereira Rosa, Alex Franco Ferreira*
}

Como citar esse artigo. Rosa, GP; Ferreira, AF. Geração de energia elétrica através de sistemas fotovoltaicos para consumidores comerciais de pequeno porte. Revista Teccen. 2019 Jul/Dez; 12 (2): 83-93.

\begin{abstract}
Resumo
Dentre outras formas de se obter energia elétrica, a solar fotovoltaica teve um crescimento superior a 30\% no Brasil por ano durante os cinco anos anteriores a 2016. Tal fato se justifica pela redução dos preços de equipamentos relacionados à geração de energia fotovoltaica, e popularização dos mesmos. O objetivo deste artigo é apresentar uma análise técnica e econômica para a instalação de sistemas fotovoltaicos de produção de energia elétrica para consumidores comerciais de pequeno porte. O local escolhido foi uma Padaria/Mercearia localizada em Mangaratiba/RJ. Para orientar a realização do estudo, é necessário seguir as normas vigentes relacionadas, REN 482 ANEEL, REN 414 ANEEL, ABNT NBR 5410, RECON BT da LIGHT, e PRODIST. Foi encontrado ao final o valor de R\$ 44.081,19 de custo total do sistema. Concluiuse, portanto, que o tempo de retorno financeiro do investimento é de 3,9 anos, levando-se em consideração que a vida útil média de um sistema fotovoltaico é de 25 anos. Chegou-se ao valor economizado de R\$282.931,28 ao longo desse período. Não foram levados em consideração possíveis gastos com a manutenção dos inversores e cabos, bem como o reajuste anual feito pelas concessionárias sobre a conta de energia elétrica.

Palavras-Chave: Eficiência Energética, Sistema Fotovoltaico, Geração de Energia Elétrica.
\end{abstract}

\begin{abstract}
Among other ways to obtain electricity, photovoltaic solar grew more than $30 \%$ per year during the five years preceding 2016. This fact is justified by the reduction of the prices of equipment related to photovoltaic power generation, and their popularization. The aim of this article is to present a technical and economical analysis for the installation of photovoltaic systems for the production of electric energy for small commercial consumers. The chosen location was a bakery/ grocery store located in Mangaratiba/RJ. To guide the realization of the study, it is necessary to follow the current norms related, REN 482 ANEEL, REN 414 ANEEL, ABNT NBR 5410, RECON BT da LIGHT, and PRODIST. The value of $\mathrm{R} \$ 44,081.19$ Total cost of the system was found at the end. It was concluded, therefore, that the financial return time of the investment is 3.9 years, taking into account that the average useful life of a photovoltaic system is 25 years. The value of $\mathrm{R} \$ 282,931.28$ has been saved over this period. It was not taken into consideration possible expenses with the maintenance of inverters and cables, as well as the annual readjustment made by the concessionaires on the electricity bill..

Keywords: Energy efficiency, Photovoltaic system, Electricity generation.
\end{abstract}

\section{Introdução}

Com a implantação de um sistema solar fotovoltaico de geração de energia, o valor da conta de luz pode ser reduzido em até $95 \%$, o aumento das tarifas pela concessionária, afeta menos o consumidor, sendo também indispensável citar que conforme Lopez (2012) "A energia solar é a fonte de energia menos poluente e menos finita conhecida até o momento".
Portanto, o objetivo deste artigo é apresentar uma análise técnica e econômica para a instalação de sistemas fotovoltaicos de produção de energia elétrica do tipo conectado à rede, para consumidores comerciais de pequeno porte.

Foi utilizado como exemplo de estabelecimento para simular a instalação, a Padaria e Mercearia Casa do Pão, situado em Mangaratiba-RJ.

A partir deste estudo, estará disponível um dimensionamento que poderá ser utilizado como base

Afiliação dos autores: Universidade de Vassouras - UV, Vassouras, RJ, Brasil

*Email para correspondência: alexfferreira@gmail.com 
para uma real aplicação no estabelecimento estudado, bem como para consumidores desta categoria de consumo, trazendo para estes, possíveis ganhos financeiros, energéticos e ambientais.

$\mathrm{O}$ estudo fundamentou-se por meio de uma revisão bibliográfica, trabalhada através da problemática na qual se encaixa esta pesquisa.

Para orientar a realização do estudo, foram utilizadas como base, as normas vigentes relacionadas, RN 482 ANEEL(2012), REN 414ANEEL(2010), ABNT NBR 5410 (2004), RECON BT da LIGHT(2019), e PRODIST(2017). Os principais livros utilizados para estudo dos fundamentos teóricos do funcionamento de sistemas solares fotovoltaicos e componentes necessários para montagem de projetos foram Kalagirou (2016), e Lopez (2012).

A sequência metodológica inicia-se com a pesquisa de dados referentes à unidade consumidora em questão.

Foirealizada a verificação do grupo de atendimento (Grupo A ou B), o tipo de tarifação (Comum ou HoroSazonal), do tipo de ligação (monofásica, bifásica ou trifásica), feito o cálculo da média anual de consumo de energia elétrica, bem como a verificação da área disponível para instalação do sistema fotovoltaico. Foram utilizados para tal, as contas de energia elétrica da unidade de 12 meses consecutivos, bem como a planta arquitetônica da construção.

O segundo passo precedente ao dimensionamento, consistiu em realizar o estudo do potencial solar (horas de sol, irradiação solar) na região estudada. A quantidade de horas de sol por dia foi obtida através do Atlas Solarimétrico do Brasil e os valores de irradiação solar média em planos e âgulos distintos foram obtidos através do Centro de referência para Energia Solar e Eólica Sérgio Brito (CRESESB, 2018), por meio do software DataSun 3.0.

Concluída a fase de levantamento de dados, passou-se às etapas do dimensionamento do sistema fotovoltaico. São estas:

1. Dimensionamento do sistema solar

2. Dimensionamento do inversor de frequência

3. Cabos

4. Dimensionamento do conjunto de suportes

5. Dimensionamento do medidor bidirecional

6. Dimensionamento dos disjuntores

7. Dimensionamento do StringBox

8. Dimensionamento do DPS

Foram consultados manuais e datasheets de inversores solares, placas solares, e demais acessórios necessários aos sistemas fotovoltaicos.

Após o projeto foi feito o levantamento do custo de cada equipamento empregado. O payback foi calculado a partir da divisão do custo total estimado do sistema pelo gasto anual em energia elétrica.

\section{Sistema de tarifação}

Conforme informa a LIGHT (2018), os grupos tarifários são $\mathrm{A}$ (fornecimento de energia, em tensão, igual ou superior a $2,3 \mathrm{kV}$ ), e B, (fornecimento em tensão inferior a $2,3 \mathrm{kV}$ ou atendidas em tensão superior a 2,3 kV e faturadas neste grupo).

Para unidades do Grupo A são considerados dois valores de consumo diferentes (Ponta e Fora ponta), portanto o dimensionamento do sistema gerador deverá considerar tal diferença. Para unidades faturadas no Grupo B, considera-se o consumo total.

\section{Taxa de disponibilidade}

Conforme informa a LIGHT (2018), para cada tipo de ligação será cobrada uma taxa referente à disponibilidade de energia elétrica, ainda que não haja consumo da energia disponibilizada na rede.

\section{Tipos de sistema}

Acoplado Diretamente: O sistema solar fotovoltaico está diretamente acoplado à carga, a qual não funciona quando os módulos não estão produzindo. (Kalagirou, 2016).

Sistemas Autônomos: Utilizam armazenamento de energia com baterias. Fica disponível apenas a energia gerada pelo sistema fotovoltaico. (CRESESB, 2014)

Sistemas Conectados À Rede: O sistema é conectado na rede através de inversores. Toda energia gerada é entregue à rede. A energia consumida vem da concessionária. (CRESESB, 2014). O medidor bidirecional lê ambos os fluxos. Ao fim de cada ciclo de faturamento, são gerados créditos ou débitos de energia. (REN 482 ANEEL, 2012)

\section{Funcionamento da célula fotovoltaica}

De acordo com Cássio Araújo do Nascimento (2004) o funcionamento da célula fotovoltaica acontece da seguinte forma:

\footnotetext{
Separadamente, ambas as capas são eletricamente neutras. Mas ao serem unidas, na região P-N, forma-se um campo elétrico devido aos elétrons livres do silício tipo $\mathrm{N}$ que ocupam os vazios da estrutura do silício tipo P. Ao incidir luz sobre a célula fotovoltaica, os fótons chocam-se com outros elétrons da estrutura do silício fornecendo-lhes energia e transformando-os em condutores. Devido ao campo elétrico gerado pela junção P-N, os elétrons são orientados e fluem da camada "P" para a camada "N". Por meio de um condutor externo, ligando a camada negativa à positiva, gera-se um fluxo de elétrons (corrente elétrica). Enquanto a luz incidir na célula, manter-se- á este fluxo. A intensidade
} 
da corrente elétrica gerada variará na mesma proporção conforme a intensidade da luz incidente. Uma célula fotovoltaica não armazena energia elétrica. Apenas mantém um fluxo de elétrons estabelecidos num circuito elétrico enquanto houver incidência de luz sobre ela. Este fenômeno é denominado "Efeito fotovoltaico". (Nascimento, 2004).

\section{Tipos de ligações dos painéis fotovoltaicos}

Ligação em Série: ao associarmos os painéis em série, as tensões dos painéis serão somadas, enquanto a corrente permanecerá a mesma, obedecendo a Lei de $\mathrm{Ohm}$

Ligação em Paralelo: ao associarmos os painéis em paralelo, a tensão permanecerá a mesma, enquanto a corrente aumentará, obedecendo a Lei de Ohm.

Os arranjos de painéis solares chamam-se string, independentemente de estarem ligados em série ou paralelo.

\section{Inversores de frequência}

Lopez (2012), define o inversor de frequência como um dispositivo eletrônico que converte a energia gerada pelas placas fotovoltaicas e/ou armazenadas pelo banco de baterias em $\mathrm{CC}$ em energia a ser consumida pelos aparelhos eletrônicos da residência em C.A.

\section{Dados da unidade consumidora}

A Padaria/mercearia estudada, tem aproximadamente $143 \mathrm{~m}^{2}$ de área disponível para construção do sistema,tarifada no Grupo B - Comercial, com ligação trifásica, situada em Mangaratiba/RJ.

O consumo médio mensal de energia é de 1027,8 KWh. Para a realização do cálculo, analisou-se o consumo de 12 (doze) meses referente ao período de junho/2017 a maio/2018, como é mostrado no Quadro 1.

Quadro 1. Consumo anual.

\begin{tabular}{|c|c|c|c|c|}
\hline Ano & Mês & Kwh & \multicolumn{2}{|c|}{ Valor Conta } \\
\hline \multirow{7}{*}{2017} & Junho & 903 & RS & 917,94 \\
\hline & Julho & 782 & RS & 794,94 \\
\hline & Agosto & 890 & RS & 904,73 \\
\hline & Setembro & 880 & RS & 894,56 \\
\hline & Outubro & 967 & RS & 983,00 \\
\hline & Novembro & 835 & RS & 848,82 \\
\hline & Dezembro & 1258 & RS & $1.278,82$ \\
\hline \multirow{5}{*}{2018} & Janeiro & 1676 & RS & $1.703,74$ \\
\hline & Fevereiro & 1024 & RS & $1.040,95$ \\
\hline & Março & 1599 & $\mathrm{RS}$ & $1.625,46$ \\
\hline & Abril & 989 & RS & $1.005,37$ \\
\hline & Maio & 530 & RS & 538,77 \\
\hline & & Média Consumo (KWh) & \multicolumn{2}{|c|}{1027,75} \\
\hline & & Média Valor Pago & RS & $1.044,76$ \\
\hline & & Total Consumo (KWh) & \multicolumn{2}{|c|}{12333} \\
\hline & & Total Valor Pago & RS & $2.537,11$ \\
\hline
\end{tabular}




\section{Localização Geográfica, Solstícios e Equinócios}

A cidade de Mangaratiba-RJ, situa-se no Hemisfério Sul do Globo Terrestre, latitude $22.9162^{\circ}$ Sul e longitude $44.0390^{\circ}$ Oeste (Google Maps, 2018).

Para Pereira e Oliveira (2011), a orientação do planeta em relação ao sol tem quatro posições: dois equinócios (quando a declinação solar é nula), e dois solstícios (quando a declinação solar é de $+23,7^{\circ}$ ou $23,7^{\circ}$, dependendo do solstício). Cada posição indica uma nova estação do ano, sendo que em casa uma destas a quantidade de radiação incidente irá ser diferente em cada Hemisfério.

De acordo com dados analisados a partir da utilização do Software DataSun 3.0 do CRESESB, verifica-se que para cidades no Hemisfério Sul do
Globo com caractéristicas similares à Mangaratiba/RJ, o melhor aproveitamento será obtido com o painel na direção norte, podendo o ângulo de inclinação variar.

\section{Estudo do potencial Solar do local}

Foi utilizado o Software SunData $3.0 \mathrm{~V}$ do CRESESB (2018) para o cálculo do potencial solar da Região em questão, onde são inseridas as coordenadas geográficas do local de interesse, como mostra a Figura 1.

De acordo com o CRESESB (2018), são retornados valores de pesquisa para 3 (três) localidades. Caso a localidade de interesse esteja listado no software, um dos valores retornados será o ponto de interesse e as outras duas localidades, próximas ao ponto de interesse. Caso o ponto de interesse não esteja listado no

\section{Coordenada Geográfica}

Latitude
22.9162
Norte:
graus decimais $\left(00.00^{\circ}\right)$
graus, minutos e segundos $\left(00^{\circ} 00^{\prime} 00^{\prime \prime}\right)$
Buscar Limpar

Figura 1. Campos de inserção de coordenadas no DataSun 3.0. Fonte: CRESESB, 2018

software são retornados valores de 3 (três) localidades próximas.

Na Figura 2 observa-se a Tabela 1 retornada pelo software a partir das coordenadas fornecidas, onde são mostrados os valores de irradiação solar diária média mensal em $\mathrm{kWh} / \mathrm{m}^{2}$, mês a mês, para as três localidades próximas a referência das coordenadas geográficas. Na coluna "Distância $[\mathrm{Km}]$ ", destacada na Figura 2, é mostrada a distância em $\mathrm{Km}$ que cada localidade retornada na pesquisa, tem do ponto de interesse (ponto latitude $22,9162^{\circ} \mathrm{S}$, longitude $44,039^{\circ} \mathrm{O}$ ).

De acordo com o DataSun 3.0, o primeiro ponto é o mais próximo das coordenadas inseridas no Software, com uma distância de $2 \mathrm{Km}$. Por esse motivo, este foi o ponto escolhido para estudo de radiação solar.

Após a escolha do ponto ideal, foi realizada a análise da radiação solar para escolha do ângulo de inclinação.

Para cada ângulo escolhido, o Software projeta uma linha no gráfico de irradiação solar entre os meses de janeiro e novembro do ano. Na Figura 3, observase o gráfico de radiação solar com os resultados para as possíveis inclinações, bem como os campos para seleções dos ângulos disponíveis no programa.

A partir da avaliação do gráfico, verifica-se que o plano $0^{\circ}$ Norte, apesar de obter o maior valor de radiação solar em fevereiro e novembro, tem durante seis meses do ano valores inferiores de radiação solar, comparativamente aos demais planos. Ao realizar a análise de comparação entre as inclinações $20^{\circ}$ Norte, $25^{\circ}$ Norte, e $23^{\circ}$ Norte, esta última apresenta valores de radiação solar que menos variam em relação aos 
Localidades próximas

Latitude: $22,9162^{\circ} \mathrm{S}$

Longitude: $44,039^{\circ}$ O

\begin{tabular}{|c|c|c|c|c|c|c|c|c|c|c|c|c|c|c|c|c|c|c|c|c|c|}
\hline \multirow{2}{*}{$\#$} & \multirow{2}{*}{ Estação } & \multirow{2}{*}{ Município } & \multirow{2}{*}{ UF } & \multirow{2}{*}{ Pais } & \multicolumn{17}{|c|}{ Irradiação solar diária média [kWh $/ \mathrm{m}^{2}$.dia] } \\
\hline & & & & & Latitude $\left[{ }^{\circ}\right]$ & Longitude $\left[{ }^{\circ}\right]$ & Distância [km] & Jan & Fev & Mar & Abr & \begin{tabular}{|l|l} 
Mai & \\
\end{tabular} & Jun & Jul & Ago & Set & Out & Nov & Dez & Média & Delta \\
\hline$\square$ & Mangaratiba & Mangaratiba & RJ & BRASIL & $22,901^{\circ} \mathrm{S}$ & $44,049^{\circ} \mathrm{O}$ & 2,0 & 5,34 & 5,75 & 4,56 & 4,07 & 3,42 & 3,16 & 3,22 & 4,02 & 4,14 & 4,53 & 4,49 & 5,20 & 4,32 & 2,59 \\
\hline$\square$ & Mangaratiba & Mangaratiba & RJ & BRASIL & $22,901^{\circ} \mathrm{S}$ & $43,949^{\circ} \mathrm{O}$ & 9,4 & 45,53 & 5,92 & 4,66 & 4,16 & 3,46 & 3,18 & 3,25 & 4,07 & 4,17 & 4,62 & 4,62 & 5,38 & 4,42 & 2,74 \\
\hline$\square$ & Oceano Atlantico & Oceano Atlantico & & & $23^{\circ} \mathrm{S}$ & $44,049^{\circ} \mathrm{O}$ & 9,4 & 45,47 & 5,83 & 4,70 & 4,26 & 3,47 & 3,22 & 3,24 & 4,06 & 4,10 & 4,54 & 4,62 & 5,30 & 4,40 & 2,62 \\
\hline
\end{tabular}

Figura 2. Valores de irradiação solar diária média mensal em $\mathrm{kWh} / \mathrm{m}^{2}$, mês a mês.

Fonte: CRESESB,2018.

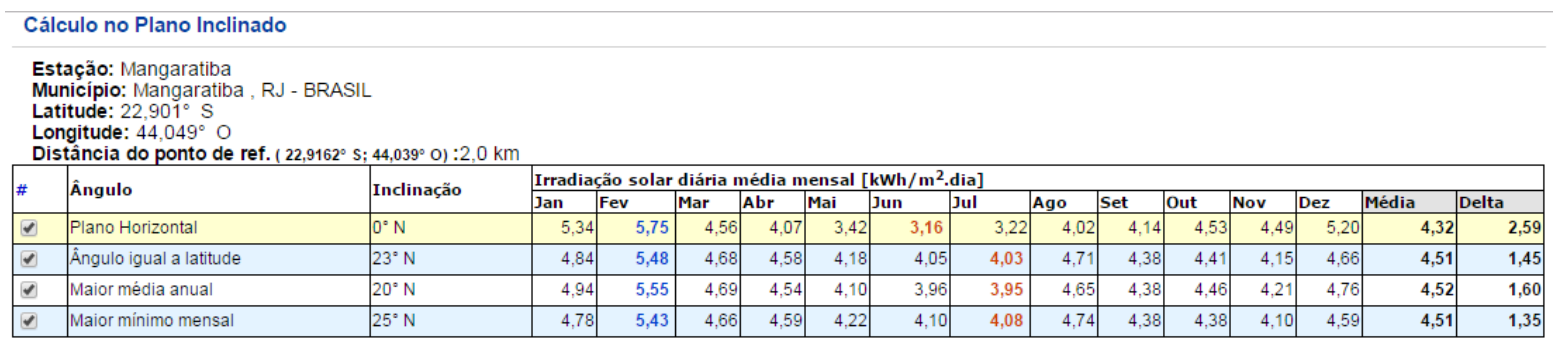

Irradiação Solar no Plano Inclinado -Mangaratiba-Mangaratiba, RJ-BRASIL

$22,901^{\circ} 5 ; 44,049^{\circ} 0$

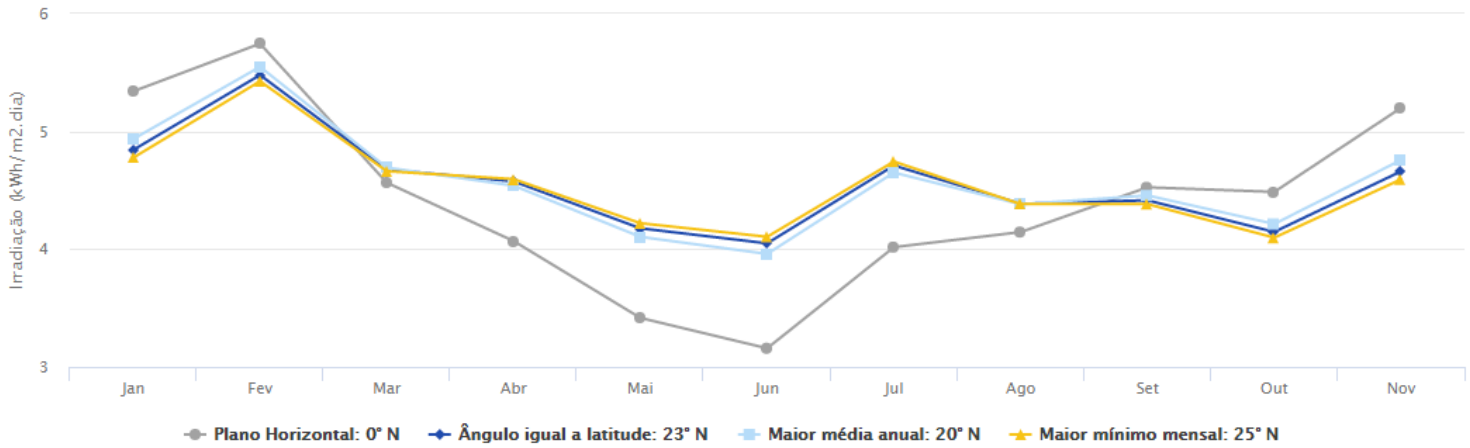

Figura 3. Valores de irradiação solar diária média mensal em $\mathrm{kWh} / \mathrm{m}^{2}$, mês a mês para ângulos distintos.

Fonte: CRESESB (2018)

demais.

Verificando a análise dos resultados obtidos através da utilização do Software SunData 3.0 do CRESESB (2018), e levando-se em consideração os estudos dos solstícios equinócios, conclui-se que a melhor orientação para instalação dos painéis solares, é o Norte Geográfico. Sendo o melhor ângulo de inclinação para instalação do módulo é de $23^{\circ}$.

\section{Dimensionamento do Sistema Solar}

\section{$1^{\circ}$ Encontrada a média do valor consumido em $\mathrm{kWh}$ por mês}

Levantou-se a média de consumo anual, que pode ser visualizada no Quadro 1. Foi utilizado o valor arredondado de $1027 \mathrm{kWh}$, e subtraída a taxa disponibilidade de energia de $100 \mathrm{kWh}$. Pode-se visualizar a operação na Equação 1:

$1027 \mathrm{kWh}-100 \mathrm{kWh}=927 \mathrm{kWh}$

\section{$2^{\circ}$ Cálculo do valor de consumo médio diário em kWh}

Encontra-se a média de consumo médio diário, dividindo-se a média de consumo mensal pela quantidade de dias do mês. Pode-se visualizar a operação na Equação 2:

$$
927 \mathrm{kWh} / 30=30,90 \mathrm{kWh} / \mathrm{dia}
$$

\section{$3^{\circ}$ Definição da quantidade de horas de sol por dia}

De acordo com o CRESESB (2000), o índice de insolação diária, por análise do mapa brasileiro na região de Mangaratiba/RJ, encontra-se em torno de 5 a 6 horas diárias, é considerado o pior caso, ou seja, 5 horas diárias.

\section{$4^{\circ}$ Rendimento do sistema}

Para dimensionar sistemas fotovoltaicos é utilizado um fator de $80 \%$ de rendimento do sistema, que considera perdas por aquecimento, sujeira, sombra. 
(Santana, 2017)

\section{$5^{\circ}$ Definição da potência necessária do conjunto}

Wp (Watt-pico) é a unidade de medida utilizada para painéis fotovoltaicos e significa a potência em W fornecida por um painel em condições especificas e reproduzidas em laboratório.Para definir a potência total do conjunto em $\mathrm{KWp}$, foi dividido o valor de consumo diário pelo produto das horas de sol por dia e do valor considerado de rendimento do sistema, de acordo com a Equação 3.

Potência $=30,90 \mathrm{KWh} /(5$ x 0,8$)$

$=7725 \mathrm{Wp}$ ou $7,725 \mathrm{KWp}$

\section{$6^{0}$ Definição do modelo de painel solar fotovoltaico}

Para concluir o dimensionamento é feita a escolha do painel considerando os fatores confiabilidade, eficiência e menor custo. Adotou-se o painel solar fotovoltaico de material policristalino da Canadian Solar, modelo CS6U-325P , que gera até $325 \mathrm{~W}$, apresenta corrente máxima de 8,78 A e tensão máxima de $37,0 \mathrm{~V}$ em corrente contínua.

\section{$7^{0}$ Definição da quantidade de painéis no sistema}

Dividiu-se a potência necessária para o sistema, pela potência do modelo de painel solar escolhido. Encontra-se o valor de 23,76 painéis necessários no sistema de acordo com a Equação 4.

$$
\text { 7,725 KWp /325 W=23,76 painéis }
$$

Para atender a demanda necessária, arredonda-se o valor encontrado para cima, chegando ao total de 24 painéis solares no sistema.

\section{$8^{\circ}$ Potência projetada do sistema, produção diária e produção mensal}

Com 24 painéis de $325 \mathrm{~W}$ no sistema fotovoltaico, tem-se um conjunto de $7,8 \mathrm{KWp}$ de potência, multiplicando a quantidade de painéis pela potência de cada placa, conforme Equação 5. A produção diária é 31,2 KWh, sendo esta calculada multiplicando-se a potência do conjunto pelo produto das horas de sol e sol e do rendimento do sistema, conforme Equação 6. A produção mensal é calculada multiplicando-se a produção diária pela quantidade de diasno mês, sendo o valor final de $936 \mathrm{KWh} /$ mês, conforme Equação 7.

Produção diária $=7,8 \mathrm{KWp} \times(5 \times 0,8)=31,2 \mathrm{Kwh} /$ dia

Potência do conjunto $=24 \times 325 \mathrm{~W}=7,8 \mathrm{KWp}$

Produção Mensal $=31,2 \mathrm{Kwh} /$ dia x $30=936 \mathrm{KWh} /$ mês (7)

\section{Dimensionamento do Inversor de Frequência \\ $1^{\circ}$ Dados do conjunto de placas solares}

Para dimensionamento do inversor, devem ser considerados os dados do conjunto de placas solares. $\mathrm{O}$ Quadro 2 relaciona as informações das placas solares fotovoltaicas.

\section{$2^{\circ}$ Escolha do inversor}

A partir da observação das informações dos painéis foi escolhido o Inversor Fronius Primo 8.2. O Quadro 3 relaciona as informações técnicas do inversor escolhido.

\section{$3^{\circ}$ Relação inversor $x$ painel}

No Quadro 4 é mostrada a relação do inversor escolhido com o conjunto de painéis. O projeto foi desenvolvido considerando a divisão das 24 placas do sistema em dois Strings de 12 placas cada. Dessa forma, o cálculo para verificar se o inversor suporta as placas solares, foi feito considerando-se os valores obtidos a partir desta associação, dispostos no Quadro 2.

\section{Cabos}

Para interligar o sistema foram escolhidos os cabos de $4 \mathrm{~mm}^{2}$, no modelo Solar $4 \mathrm{~mm}$, e $6 \mathrm{~mm}^{2}$, no modelo 6mm + MC4 (DUARTE, 2016).

\section{Dimensionamento do conjunto de suportes}

\section{$1^{\circ}$ Escolha do tipo de suporte}

O local de instalação do conjunto fotovoltaico foi a cobertura do estabelecimento. Portanto, foi escolhido o suporte da Romagnole Modelo Solo, o qual aloja 4 placas cada, do tipo de fixação em laje, e ângulo de inclinação ajustável entre os valores de 5 e 30 graus (KASATEC, 2018). Esta característica viabiliza a montagem das placas no ângulo de $23^{\circ}$ graus, conforme definido neste artigo.

\section{$2^{\circ}$ Relação suporte $\mathrm{x}$ placas solares}

Encontra-se o número total de suportes, dividindo-se a quantidade total de placas solares, pela quantidade que cada suporte comporta, de acordo com a Equação 8.

Quantidade de suportes $=24 / 4=6$ 
Quadro 2. Dados do painel.

\begin{tabular}{|l|l|l|l|}
\hline Dados & Painel & $\begin{array}{l}\text { Dados de } \\
\text { todos os } \\
\text { painéis em } \\
\text { série }\end{array}$ & $\begin{array}{l}\text { Painéis em String } \\
\text { (Dados painéis } \\
\text { agrupados em duas } \\
\text { strings de 12 placas } \\
\text { cada) }\end{array}$ \\
\hline Potencia nominal (W) & 325 & 7800 & 7800 \\
\hline Tensão máxima (V) & 37 & 888 & 444 \\
\hline Corrente máxima de operação (Imp)(A) & 8,78 & 8,78 & 17,56 \\
\hline Tensão do circuito aberto (Voc) (V) & 45,5 & 1092 & 546 \\
\hline Corrente de Curto Circuito (Isc) (A) & 9,34 & 9,34 & 18,68 \\
\hline
\end{tabular}

Quadro 3. Dados do inversor.

\begin{tabular}{|l|l|}
\hline Dados & Inversor Fronius Primo 8.2 \\
\hline Potência CC máxima (W) & 8500 \\
\hline Tensão máxima de entrada (V) (Vmax) & 1000 \\
\hline $\begin{array}{l}\text { Faixa de Tensão MPP ( MPP: Ponto de Máxima } \\
\text { Potência) - (V) }\end{array}$ & $270-800$ \\
\hline Corrente máxima de entrada (A) (Imax) & 18 \\
\hline Potencia CA máxima (W) & 8200 \\
\hline Corrente máxima de saída (A) & 35,7 \\
\hline Tensão máxima de saída (V) & 270 \\
\hline
\end{tabular}

Fonte: Fronius, 2018.

Quadro 4. Relação inversor x painel.

\begin{tabular}{|l|l|l|l|}
\hline Dados & $\begin{array}{l}\text { Referência } \\
\text { (inversor/painel) }\end{array}$ & Cálculo & $\begin{array}{l}\text { Relação Strings } \\
\text { agrupados x } \\
\text { Inversor }\end{array}$ \\
\hline Potencia (W) & Pcc/Pnom & $8500 / 7800$ & 1,1 \\
\hline Tensão (V) & Vmppmax/V max & $800 / 444$ & 1,8 \\
\hline Tensão Máxima (V) & Vmax/ Voc & $1000 / 546$ & 1,8 \\
\hline Corrente (A) & Imax/Imp & $18 / 17,56 \quad$ Fonte: Própria, 2018. \\
\hline
\end{tabular}




\section{Dimensionamento do medidor Bidirecional}

Adotou-se para o projeto o medidor trifásico bidirecional de modelo E34A da Landis Gyr. O medidor atende tensões de entrada de 120 e $240 \mathrm{~V}$, com corrente nominal de $15 \mathrm{~A}$ e corrente máxima de $120 \mathrm{~A}$. A classe de frequência é de 50 e $60 \mathrm{~Hz}$.

\section{Dimensionamento dos disjuntores}

Para dimensionamento dos disjuntores de proteção das placas solares, foi multiplicada a corrente de saída dos Strings por um fator de segurança de 20\% (Duarte, 2016), de acordo com a Equação 9. Foram adotados para proteção dos Strings disjuntores bipolares de $25 \mathrm{~A}$ do Modelo K32a2B25 da Schneider.

$$
17,56 \mathrm{~A} \times 1,20=21,1
$$

Para dimensionamento do disjuntor de proteção instalado na saída do inversor, foi multiplicada a corrente de saída do inversor por um fator de segurança de $20 \%$ (Duarte, 2016), conforme a Equação 10. Foi adotado um disjuntor bipolar 50 A do Modelo K32a2B50 daSchneider.

$$
35,7 \mathrm{~A} \times 1,20=42,8 \mathrm{~A}
$$

\section{Dimensionamento do String Box}

De acordo com a CLAMPER (2019), a String Box, é responsável por receber as conexões dos arranjos das placas fotovoltaicas,realizar a proteção contra raios e surtos elétricos e seccionar o circuito entre as placas

Quadro 5. Características do String Box.

\begin{tabular}{|l|l|l|}
\hline Característica & Tensão (V) & Corrente (A) \\
\hline DPS & $1000 \mathrm{~V}$ & $40 \mathrm{kA}$ \\
\hline Chave seccionadora & $1000 \mathrm{~V}$ & $25 \mathrm{~A}$ \\
\hline Grau de proteção & IP65 \\
\hline
\end{tabular}

Fonte: Mercado Livre, 2018

fotovoltaicas e o inversor. Adotou-se o StringBox ABB STR 25 A 1000 V, com características básicas dispostas no Quadro 5.

\section{Dimensionamentos do DPS (Dispositivo de Proteção de Surto)}

De acordo com a CLAMPER (2012), DPS é a sigla para Dispositivo de Proteção contra Surtos. Aparelhos capazes de proteger equipamentos eletroeletrônicos contra picos de tensão. O DPS utilizado no projeto será de classe 2. O DPS classe 2 é destinado a proteção contra surtos elétricos ocasionados por descargas atmosféricas indiretas, ou seja, caem próximo à edificação ou as linhas de transmissão de energia ou dados. (CLAMPER, 2019). O DPS adotado será de $20 \mathrm{kA}$, do modelo 5SD7 461-0 MB da Siemens, como mostrado no Quadro 6.O modelo de aterramento executado é o TN-C, onde as funções do neutro e do condutor de proteção são combinadas um único condutor ao longo de toda a instalação (Niskier
\& Macintyre, 2000). A densidade de descargas está em torno de 12 por $\mathrm{km}^{2} /$ ano para a região em questão.

\section{Análise Estrutural - Área}

Amodelo deplacas escolhidomede $992 \mathrm{~mm} \times 1960$ $\mathrm{mm}$. Os Strings ocupam portando, aproximadamente $47 \mathrm{~m}^{2}$. Considerando que a área disponível na unidade consumidora é de aproximadamente $143 \mathrm{~m}^{2}$, tem-se área para montagem do sistema fotovoltaico.

\section{Análise Financeira}

No Quadro 7 visualiza-se o custo estimado do sistema.

\section{Gasto Atual com Energia Elétrica}

De junho/2017 a maio/2018 foram gastos 12333 KWh de energia, totalizando uma valor pago de R\$ 
Quadro 6. Dados do DPS.

\begin{tabular}{|l|l|l|}
\hline Execução & $\begin{array}{l}\text { Corrente nominal } \\
\text { de descarga }\end{array}$ & $\begin{array}{l}\text { Corrente nominal de } \\
\text { descarga máxima }\end{array}$ \\
\hline 1 polo (TN-C) & $20 \mathrm{kA}$ & $40 \mathrm{kA}$ \\
\hline
\end{tabular}

Fonte: Mercado Livre, 2018.

Quadro 7. Custo estimado do sistema.

\begin{tabular}{|c|c|c|c|}
\hline Material & $\begin{array}{l}\text { Quantidade } \\
\text { (unidade) }\end{array}$ & Valor Unitário (RS) & Valor Total (RS) \\
\hline Painéis & 24 & RS 730,00 & RS $17.520,00$ \\
\hline Inversor & 1 & RS $14.159,00$ & RS $14.159,00$ \\
\hline Cabo $6 \mathrm{~mm}^{2}$ (Kit $\left.10 \mathrm{~m}\right)$ & 1 & RS 149,90 & RS $\quad 149,90$ \\
\hline Cabo $4 \mathrm{~mm}^{2}$ (Kit $\left.30 \mathrm{~m}\right)$ & 1 & $\begin{array}{ll}\text { RS } & 179,00\end{array}$ & RS 179,00 \\
\hline Kit de estrutura & 6 & RS $1.672,00$ & RS $10.032,00$ \\
\hline Medidor & 1 & 308,49 & 308,49 \\
\hline Disjuntor $25 \mathrm{~A}$ & 2 & 28,62 & 57,24 \\
\hline Disjuntor $50 \mathrm{~A}$ & 1 & 38,00 & 38,00 \\
\hline String Box & 1 & RS $1.450,00$ & RS $1.450,00$ \\
\hline DPS & 1 & 187,56 & RS $\quad 187,56$ \\
\hline Custo total & - & - & RS $44.081,19$ \\
\hline
\end{tabular}

Fonte: Própria, 2018.

12.537,11 como mostrado no Quadro 1 desse trabalho. Para encontrar a parcela referente a disponibilidade de energia, multiplica-se o valor do KWh, que é 1,02 reais, por 100 (valor em KWh pago pelos consumidores com ligação trifásica para disponibilidade de energia), de acordo com a Equação 11.

$$
\mathrm{R} \$ 1,02 \times 100=\mathrm{R} \$ 101,66
$$

Para encontrar o valor pago de junho/2014 a maio/2018, multiplica-se o valor mensal por 12 , de acordo com a Equação 12.

$$
\mathrm{R} \$ 101,66 \times 12=\mathrm{R} \$ 1219,86
$$

A disponibilidade de energia continuará sendo paga mesmo após a implementação do projeto, logo o valor real economizado anual é de $\mathrm{R} \$ 11.317,25$, como mostra a Equação 13.

$$
\begin{aligned}
& \mathrm{R} \$ 12.537,11-\mathrm{R} \$ 1219,86=\mathrm{R} \$ \\
& 11.317,25
\end{aligned}
$$

\section{Retorno de investimento}

Dividindo o custo estimado do sistema pelo gasto anual em energia (valor utilizado considera o gasto que será eliminado, não contando portanto, a taxa de disponibilidade), chega-se a Equação 14, que apresenta o tempo de retorno do sistema:

$$
\mathrm{R} \$ 44.081,19 / \mathrm{R} \$ 11.317,25=3,9 \text { anos }
$$

Os possíveis aumentos na conta de energia, relacionados à taxação por bandeiras tarifárias, são 
desconsiderados neste cálculo.

\section{Projeção de valores em 25 anos}

Considerando que a vida útil média dos módulos é de 25 anos (Duarte, 2016), pode-se fazer a projeção de valor que deixará de ser pago à concessionária de energia neste tempo. Para realização do cálculo foi considerado que a taxa de disponibilidade continuará sendo paga, e que o valor economizado anual é de $\mathrm{R} \$ 11.317,25$, e não foram levados em consideração possíveis gastos com a manutenção dos inversores e cabos, bem como o reajuste anual feito pelas concessionárias sobre a conta de energia elétrica. Dessa forma, a economia na faixa de 25 anos pode ser vista na Equação 15.

$$
25 \times \mathrm{R} \$ 11.317,25=\mathrm{R} \$ 282.931,28
$$

\section{Considerações finais e conclusão}

O projeto mostra-se tecnicamente viável, altamente vantajoso economicamente, devido ao significativo valor economizado e baixo payback, além de ser uma alternativa sustentável para gerar energia elétrica.

Para meses em que a geração exceder o valor de energia consumida, serão gerados créditos de energia que poderão ser utilizados posteriormente.

A partir do levantamento dos aspectos relacionados à localização, bem como ao padrão de consumo da unidade consumidora estudada, foi possível realizar o dimensionamento dos componentes do sistema solar fotovoltaico responsável pela geração de energia elétrica.

Apósarealizaçãodoscálculosdedimensionamento dos componentes foi encontrado o valor de $\mathrm{R} \$ 44.081,19$ de custo total do sistema. Concluiu-se, portanto, que o tempo de retorno financeiro do investimento é de 3,9 anos. Levando-se em consideração que a vida útil média de um sistema fotovoltaico é de 25 anos, chegou-se ao valor economizado de $\mathrm{R} \$ 282.931,28$ ao longo desse período.

\section{Referências bibliográficas}

ABB (2018). Quadro Protetor De Surto String Box 25A 1000V - Abb. Retirado 12, 2018, de https://sicessolar.com.br/PDF/ABBStringBox/1.0STR INGBOXABB1OU2CORDA1SAIDA/1SLM300101A0790BR.pdf.

ABNT NBR 5410 (2004). Instalações Elétricas de Baixa Tensão. Retirado 08, 2019, de https://www.abnt.org.br/.

ANEEL (2017). Procedimentos de Distribuição de Energia Elétrica no Sistema Elétrico Nacional: Módulo 3 - Acesso ao Sistema de Distribuição. Retirado 03, 2018, de http://www.aneel.gov.br/modulo-3.

ANEEL (2010). Resolução Normativa $N^{o}$ 414, De 9 De Setembro De 2010:
Estabelece as Condições Gerais de Fornecimento de Energia Elétrica de forma atualizada e consolidada. Retirado 12, 2019, de https://www.aneel. gov.br/documents/656877/14486448/bren2010414.pdf/3bd33297-26f94ddf-94c3-f01d76d6f14a?version=1.0.

ANEEL (2012). Resolução Normativa $N^{o}$ 482. Retirado 03, 2018, de http:// www2.aneel.gov.br/cedoc/ren2012482.pdf.

Canadian (2018). Datasheet Painel 325 W Canadian Solar. Retirado 10, 2018, dehttps://recursos.minhacasasolar.com.br/MediaCenter/Datasheet $\% 20$ Canadian\%20Solar\%20325W\%20em\%20Ingl\%C3\%AAs.pdf.

CLAMPER (2012). DPS. Retirado 12, 2019, de https://www.clamper.com. br/2012/08/30/afinal-o-que-e-um-dps.

CLAMPER (2019). Quais as classes do DPS. Retirado 12, 2019, de https:// www.clamper.com.br/faq/quais-as-classes-do-dps/.

CLAMPER (2019). String Box: A proteção para o seu sistema fotovoltaico. Retirado 12, 2019, de https://www.clamper.com.br/2019/10/07/clamperstringbox/.

CRESESB (2000). Atlas Solarimétrico do Brasil. Retirado 03, 2018, de http://www.cresesb.cepel.br/publicacoes/download/Atlas_Solarimetrico do_Brasil_2000.pdf.

CRESESB (2008). Radiação Solar: Captação e Conversão. Retirado 05, 2018, de http://www.cresesb.cepel.br/index.php?section=com content\&lang=pt\&cid=301.

CRESESB (2014). Componentes de um sistema fotovoltaico. Retirado 03, 2018, de http://www.cresesb.cepel.br/index.php?section=com content\&lang $=$ pt\&cid $=341$.

CRESESB (2018). Potencial Solar - SunData v 3.0. Retirado 07, 2018, de http://www.cresesb.cepel.br/index.php?section=sundata\&.

Duarte, A. P. B. S. (2016). Geração De Energia Elétrica Através De Sistemas Fotovoltaicos Para Pequenos Consumidores. (TCC - Graduação) Universidade de Vassouras.

FRONIUS (2018). Datasheet Inversor Solar Fronius. Retirado 10, 2018, de https://recursos.minhacasasolar.com.br/MediaCenter/Datasheet-InversorFronius-Primo-em-Portugu\%C3\%AAs.pdf.

GOOGLEMAPS (2018). Localização de Mangaratiba-RJ. Retirado 05, 2018, de https://www.google.com.br/maps/@-22.9721002,-44.0975675,12z.

Kalagirou, S. A. (2016). Engenharia de Energia Solar: processos e sistemas. (2. ed., Vol 1) Rio de Janeiro, Rio de Janeiro. Elsevier Editora

KASATEC (2018). Estrutura de Fixação para 4 placas. Retirado 10, 2018, de http://www.kasatec.com.br/manuais/Estrutura_Solo.pdf.

LANDIS GYR (2019). Medidor De Energia Elétrica Trifásico Landis Gyr E34a. Retirado 12, 2019, de https://www.landisgyr.com.br/webfoo/wpcontent/uploads//2012/12/LandisGyr_E34A.pdf .

LIGHT (2016). Procedimentos para a Conexão de Microgeração e Minigeração ao Sistema de Distribuição da Light SESA BT e MT - Até Classe 36,2kV. Retirado 06, 2018, de http://www.light.com.br/Repositorio/ Recon/LIGHT_Informacao_Tecnica_DTE_DTP_01_2012_MARCO_2016. pdf.

LIGHT (2018). Grupos Tarifários. Retirado 05, 2018, de http://www.light. com.br/para-empresas/Tarifas-e-Tributos/grupos-tarifarios.aspx.

LIGHT (2019). RECON BT - Entradas Individuais e Coletivas: Regulamentação para fornecimento de energia elétrica a consumidores em baixa tensão. Retirado 12, 2019, de http://www.light.com.br/Repositorio/ Recon/RECON-BT-COMPLETO.pdfLOPEZ, Ricardo Aldabó. Energia solar para produção de eletricidade. Rio de Janeiro: Liberj, 2012.

Lopez, R. A. (2012). Energia solar para produção de eletricidade. (1. ed. , Vol 1) Rio de Janeiro, Rio de Janeiro. Artiliber.

Mercado Livre (2018). DPS Classe 2. Retirado 10, 2018, de https://produto. mercadolivre.com.br/MLB-803860335-dps-classe-2-dispositivo-protetorde-surto-2040ka-siemens-_JM.

Mercado Livre (2018). Quadro Protetor De Surto String Box 25a 1000v - Abb. Retirado 10, 2018, de https://produto.mercadolivre.com.br/MLB- 
997692232-quadro-protetor-de-surto-string-box-25a-1000v-abb-_JM.

Nascimento, C. A. (2004). Princípio De Funcionamento Da Célula Fotovoltaica. Retirado 10, 2018, de http://www.solenerg.com.br/files/ monografia_cassio.pdf.

Niskier, J. \& Macintyre, A. J. (2000). Instalações Elétricas (4 ed., Vol. 1, pág. 1-550). Rio de Janeiro, Rio de Janeiro: LTC - Livros Técnicos e Científicos SA

Pereira, F. A. de S. \& OLiveira, M. A. S. (2011). Curso Técnico Instalador de Energia Solar Fotovoltaica. (2. Ed, Vol.1 ). Porto, Portugal. Publindústria, Edições Técnicas

Santana, L. S. (2017). Quantos Painéis Solares Preciso para uma Residência. Retirado 10, 2018, de https://blog.bluesol.com.br/quantos-paineis-solarespreciso-para-uma-residencia/.

SCHNEIDER (2019). Disjuntores Modulares. Retirado 12, 2019, de https:// tools.schneider-electric.com.br/prime/produtos/protecaoeletrica/disjuntores/ pdf/Disjuntores.pdf.

SIEMENS (2019). Dispositivos de proteção contra surtos - DPS. Retirado 08, 2019, de http://www.solcentral.com.br/wp-content/uploads/2016/04/ Siemens_DPS_pt-br.pdf. 